Baltic Astronomy, vol. 24, 305-313, 2015

\title{
STRUCTURAL PARAMETERS OF STAR CLUSTERS: SIGNAL TO NOISE EFFECTS
}

\author{
D. Narbutis, A. Bridžius and D. Semionov \\ Center for Physical Sciences and Technology, \\ Savanoriu 231, Vilnius LT-02300,Lithuania; donatas.narbutis@ftmc.lt
}

Received: 2015 September 28; accepted: 2015 October 15

\begin{abstract}
We study the impact of photometric signal to noise on the accuracy of derived structural parameters of unresolved star clusters using MCMC model fitting techniques. Star cluster images were simulated as a smooth surface brightness distribution following a King profile convolved with a point spread function. The simulation grid was constructed by varying the levels of sky background and adjusting the cluster's flux to a specified signal to noise. Poisson noise was introduced to a set of cluster images with the same input parameters at each node of the grid. Model fitting was performed using "emcee" algorithm. The presented posterior distributions of the parameters illustrate their uncertainty and degeneracies as a function of signal to noise. By defining the photometric aperture containing $80 \%$ of the cluster's flux, we find that in all realistic sky background level conditions a signal to noise ratio of $\sim 50$ is necessary to constrain the cluster's half-light radius to an accuracy better than $\sim 20 \%$. The presented technique can be applied to synthetic images simulating various observations of extragalactic star clusters.
\end{abstract}

Key words: galaxies: star clusters: general, techniques: image processing

\section{INTRODUCTION}

We consider the simplest case of an unresolved star cluster detected with an ideal CCD in a single image and located in a completely empty environment. The only sources of photons are the cluster and uniform sky background. Let the point spread function (PSF) be known. We construct the cluster's model at position $(x, y)$ in the image by using a King (1962) surface brightness profile defined by a core radius, $r_{\mathrm{c}}$, and a tidal radius, $r_{\mathrm{t}}$. We convolve it with the PSF, multiply by the cluster's flux, $f_{\text {cl }}$, and add sky background, $\mu_{\text {sky }}$. This model image is compared to the observed image to evaluate the goodness of the model. The sky background usually is determined from pixels surrounding the cluster and subtracted before model fitting. However, we treat $\mu_{\text {sky }}$ as free and perform complete analysis of the recovered parameters.

The questions we address in this paper are: 1) what is the accuracy of derived parameters $\left(x, y, r_{\mathrm{c}}, r_{\mathrm{t}}, f_{\mathrm{cl}}, \mu_{\mathrm{sky}}\right)$ ? 2) How this accuracy depends on the photometric signal to noise? 3) How degenerate are these parameters? 4) What is the minimal signal to noise required to constrain the cluster's half-light radius $r_{\mathrm{h}}$ ?

For simplicity, we do not take into consideration the stochastic effects due 
to random sampling of stellar mass function. The stochasticity of bright stars is significant for low mass star clusters, therefore, the results of this study are primarily applicable to massive clusters. The completely empty sky background is realistic for the clusters in galaxy halos. However, even for bright clusters projected on the galaxy we can assume that an unresolved background stellar population is a uniform sky background with elevated $\mu_{\text {sky }}$ level.

\section{IMAGE SIMULATION}

We assume King (1962) surface brightness profile defined by its central value, $\mu_{0}$, a core radius, $r_{\mathrm{c}}$, and a tidal radius, $r_{\mathrm{t}}$ :

$$
\mu(r)=\mu_{0}\left[\left(1+\frac{r^{2}}{r_{\mathrm{c}}^{2}}\right)^{-1 / 2}-\left(1+\frac{r_{\mathrm{t}}^{2}}{r_{\mathrm{c}}^{2}}\right)^{-1 / 2}\right]^{2}
$$

For further analysis we consider clusters with $r_{\mathrm{c}}=3$ pix and $r_{\mathrm{t}}=30$ pix, which are extended objects with shallow surface brightness profiles and affected by noise more than compact clusters. Pixel values of a $2 \mathrm{D}$ array were computed using Eq. 1 by placing a cluster in the center of the image, however, allowing its position to be randomized within the pixel. The image is square with a side length of $2 r_{\mathrm{t}}+1=61$ pix. It was convolved with a Gaussian PSF of FWHM $=3$ pixels. The total flux of the cluster was set to $f_{\mathrm{cl}}$. A constant sky background, $\mu_{\mathrm{sky}}$, was added and Poisson noise was introduced to each pixel to simulate observations.

We define the photometric aperture of radius $r_{\text {phot }}=10$ pix, which, for the chosen $r_{\mathrm{c}}=3$ and $r_{\mathrm{t}}=30$ pix and the PSF, integrates $80 \%$ of the cluster's total flux. We denote this flux as $f_{\text {phot }}=0.8 f_{\mathrm{cl}}$ and compute the signal to noise ratio within the aperture following Newberry (1991):

$$
S / N=\frac{f_{\mathrm{phot}}}{\sqrt{f_{\mathrm{phot}}+\pi \cdot r_{\mathrm{phot}}^{2} \cdot \mu_{\mathrm{sky}}}} .
$$

The sky background level depends on the exposure time, telescope size, passband, etc. We analyze three cases of the sky background levels having flux ratios 1:10:100, which correspond to the the flux ratios in galaxy halo, disk, and bulge, i.e., weak, moderate, and strong. Therefore, the background levels were set to $\mu_{\text {sky }}$ $=10^{2}, 10^{3}$, and $10^{4} \mathrm{ADU}$ (photons; inverse gain $=1 \mathrm{e}^{-} / \mathrm{ADU}$ ).

We have chosen to investigate the cases $S / N=5,10,20,50,100,200,500$, and 1000 for each case of the sky background level by adjusting the cluster's flux, $f_{\mathrm{cl}}$, to achieve the required $S / N$. We have simulated the cluster grid with three sky and eight signal to noise values; 24 nodes in total. For each node of the grid, 100 cluster images were analyzed.

Examples of simulated clusters placed in sky background of $\mu_{\text {sky }}=10^{3}$ ADU are displayed in Fig. 1 for eight cases of $S / N$. The $S / N=5$ cluster is not detectable by eye, while that with $S / N=10$ is barely distinguishable. Progressing to $S / N$ $=50$, the object is more prominent and suitable for model fitting. However, the pixel values beyond the photometric aperture $r_{\text {phot }}=10$ pix, shown as the inner circle, are dominated by noise even for the $S / N=200$ cluster. When the $S / N$ $=1000$ cluster is considered, which is close to the maximum $S / N$ achievable in a single exposure before the core of the cluster gets saturated, pixels close to the 


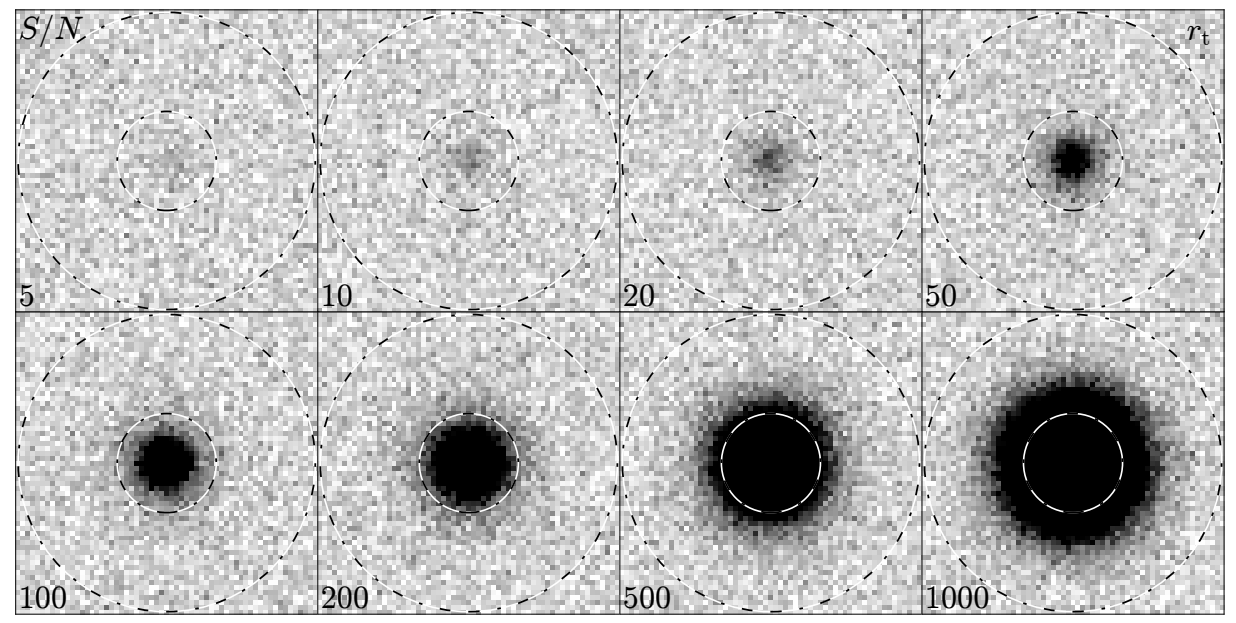

Fig. 1. Examples of simulated star clusters placed in the sky background $\mu_{\text {sky }}=$ $10^{3}$ ADU. The signal to noise $(S / N)$ values $(5,10,20,50,100,200,500$, and 1000) are displayed in the panels. Inner circle of $r_{\text {phot }}=10$ pix indicates the photometric aperture used to define the $S / N$ within it. Outer circle indicates the tidal radius $r_{\mathrm{t}}=30$ pix $\left(r_{\mathrm{c}}\right.$ $=3$ pix). Grey levels correspond to pixel values from 950 to $1250 \mathrm{ADU}$ in linear scale. The cluster's center with $S / N=1000$ has a value of $\sim 41000$ ADU.

tidal radius $r_{\mathrm{t}}=30$ pix, shown as the outer circle, are still dominated by the noise. In this model (Eq. 1), pixels beyond $r_{\mathrm{t}}$ contain photons from the sky background only.

The other cases of the sky background levels, $\mu_{\text {sky }}=10^{2}$ and $10^{4}$ ADU, show the results visually similar to those in Fig. 1, however, when the sky level is high, it is a dominant source of uncertainty even for brighter clusters.

\section{MODEL FITTING}

To perform star cluster analysis we use the Markov Chain Monte Carlo (MCMC) model fitting technique implemented in the "emcee" algorithm (Foreman-Mackey et al. 2013). The model parameters are: 1) position, $(x, y), 2)$ core and tidal radii, $r_{\mathrm{c}}$ and $\left.r_{\mathrm{t}}, 3\right)$ cluster's total flux, $f_{\mathrm{cl}}$, and 4) sky background level, $\mu_{\mathrm{sky}}$. To reduce computation time, we initialize the "emcee" with true parameters of the simulated cluster. The "emcee" starts a number of independent Markov chains which sample the parameter space according to the goodness criterion, which is the probability, $P$, that the observed image can be explained by the model with given parameters.

We compute the probability of the model, $P$, by multiplying the prior probability and the likelihood. We assume a flat prior probability for the following parameter ranges: 1) position ( $x, y)$ within 3 pixels of true position, 2) $0.1<r_{\mathrm{c}}<r_{\mathrm{t}}<200$ pix, 3) $10<f_{\mathrm{cl}}<10^{9} \mathrm{ADU}$, 4) $1<\mu_{\mathrm{sky}}<10^{5}$ ADU. If the Markov chain of " $\mathrm{em}$ cee" reaches the boundary values, the prior probability 0 is returned and, therefore, the probability of the model $P=0$. This allows the model to be defined within reasonable limits of the parameter values, where the probability of the model is equal to the likelihood of the model. We build a smooth cluster model (Eq. 1) plus sky background as described in the previous section to compute the likelihood.

To estimate model fitting uncertainties due to photon noise, we consider the 
observed image and assume that for a pixel with flux $\mu_{\mathrm{obs}}$ its uncertainty is $\sqrt{\mu_{\mathrm{obs}}}$ and is equal to the standard deviation of the Gaussian distribution. This assumption is reasonably applicable even for simulated images with $\mu_{\text {sky }}=10^{2}$ ADU with Poisson noise. Computation of the likelihood assuming a Gaussian distribution of uncertainty is much faster than assuming a Poisson distribution.

We compute the Gaussian likelihood of the data pixel, given the model pixel. We multiply the likelihoods of pixels assuming that they are independent and assign the likelihood to the probability $P$ because of a flat prior. The "emcee" uses $\ln (P)$ since the numerical values of probability are small when dealing with a large number of pixels. All pixels in the image are used for computation. Pixels in the corners of the image are free of the cluster's flux and allow to constrain the sky background as an independent parameter.

We run the "emcee" for 3000 steps as a "burn-in" phase while Markov chains reach the vicinity of the maximum likelihood position in the parameter space. We drop these steps and run for 5000 steps more, which sample the posterior values of the model parameters. These posterior distributions are analyzed in the following section.

\section{RESULTS AND DISCUSSION}

We analyze the results of "emcee" model fitting by producing corner plots, which show all the one- and two-dimensional projections of the posterior probability distributions of the parameters $\left(x, y, r_{\mathrm{c}}, r_{\mathrm{t}}, f_{\mathrm{cl}}, \mu_{\mathrm{sky}}\right)$, and demonstrate all the covariances between the parameters and their marginalized distributions in histograms.

In Fig. 2 we show the posterior distributions of $r_{\mathrm{c}}, r_{\mathrm{t}}, f_{\mathrm{cl}}$, and $\mu_{\mathrm{sky}}$ for the $S / N=20$ cluster displayed as black contours. True values are indicated by an intersection point of large cross-bars. Open circles show median values of the recovered parameter distributions of 100 simulated clusters with the same input parameters, but different randomization of Poisson noise. The radius $r_{\mathrm{c}}$ is recovered reasonably well, however, judging by concentration of open circles at $r_{\mathrm{t}} \sim 100$ pix (middle of $r_{\mathrm{t}}$ prior range), we conclude that the $S / N=20$ is too low to constrain the tidal radius. Non-linear degeneracies between the parameters, e.g., $r_{\mathrm{c}}$ and $r_{\mathrm{t}}$, are observed. The sky background is underestimated and the cluster's flux is overestimated in the majority of cases.

When a cluster with larger $S / N$ is considered, see Fig. 3 for the case $S / N$ $=50$, the parameter degeneracies become almost linear and histograms have less extended tails. The degeneracy between $r_{\mathrm{c}}$ and $r_{\mathrm{t}}$ is prominent because models with small $r_{\mathrm{c}}$ and large $r_{\mathrm{t}}$ are as likely as models with large $r_{\mathrm{c}}$ and small $r_{\mathrm{t}}$. In the outskirts of the cluster, which are dominated by the sky background noise, models with large $r_{\mathrm{t}}$ are as likely as models with small $r_{\mathrm{t}}$, but there is also a strong degeneracy between $r_{\mathrm{t}}$ and $f_{\mathrm{cl}}$, because models with larger extent have a larger integral flux.

Although beyond $r_{\mathrm{t}}$ photons only from the sky background are detected, the majority of pixels in the image are within $r_{\mathrm{t}}$, and the sky background is determined within the cluster's area. Therefore, $\mu_{\text {sky }}$ is also degenerate with $r_{\mathrm{t}}$, because when a model with larger $r_{\mathrm{t}}$ is considered, $\mu_{\mathrm{sky}}$ must be smaller then the true value in order to compensate for the excess flux. The uncertainty in the sky background remains identical to that in the $S / N=20$ case, because it is determined primarily 


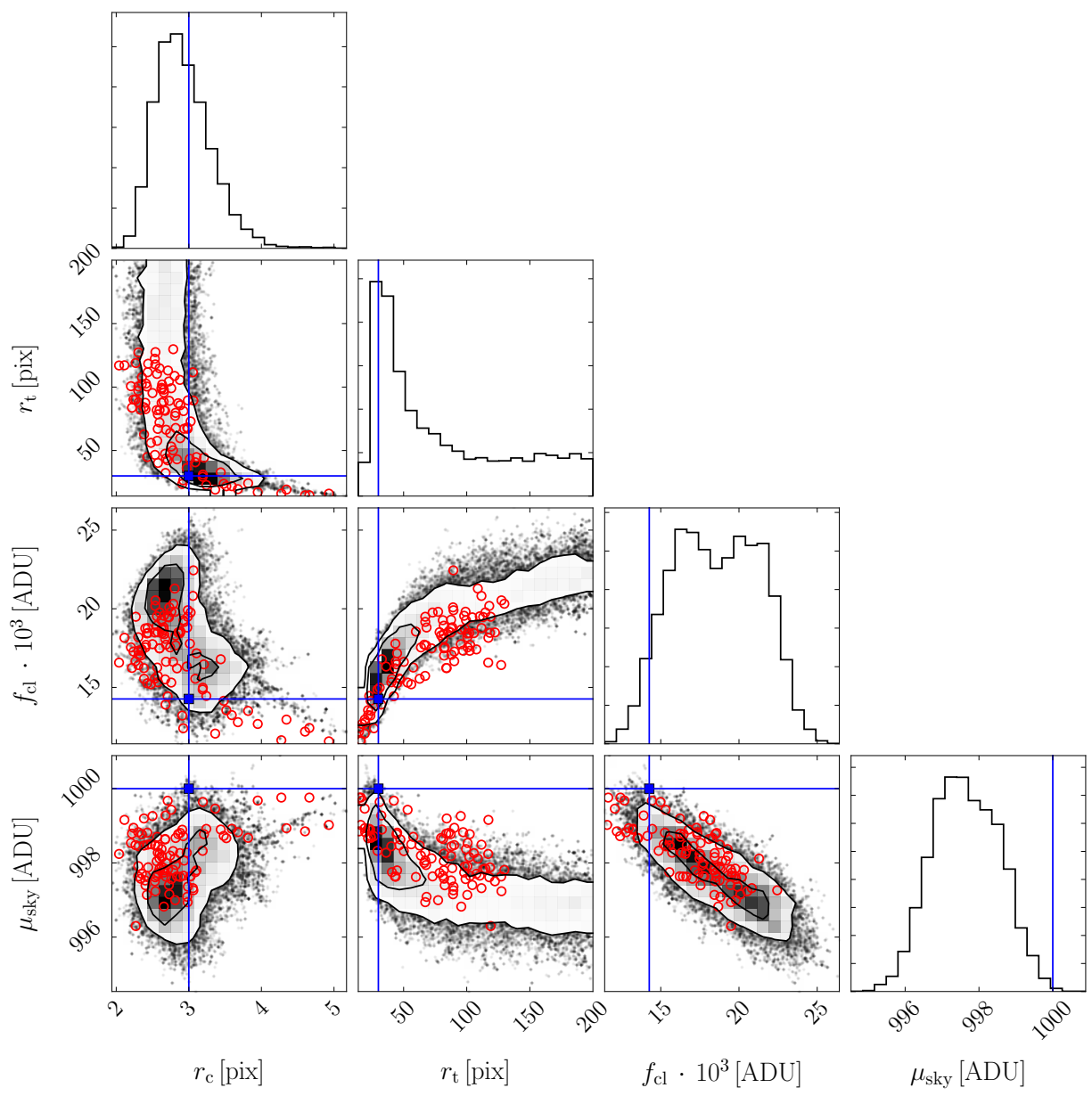

Fig. 2. Posterior distributions of core, $r_{\mathrm{c}}$, and tidal, $r_{\mathrm{t}}$, radii, flux $f_{\mathrm{cl}}$, and sky background $\mu_{\text {sky }}$, shown as histograms and two parameter covariance plots for a star cluster with $S / N=20$ and $\mu_{\text {sky }}=10^{3}$ ADU. Contours are shown at 1- and 2- $\sigma$ levels. True parameter values are indicated by an intersection of large cross-bars. Open circles show median values of the recovered parameter distributions of 100 simulated clusters with the same input parameters but different randomization of Poisson noise.

by the number of pixels that are unaffected by the cluster's flux $\left(r>r_{\mathrm{t}}\right)$.

For a cluster with $S / N=100$ (Fig. 4), the parameter degeneracies are more pronounced, but parameter uncertainties are smaller. Judging from the posterior distributions of the parameters of a single cluster, displayed as contours in Figs. $2-4$, and the distributions of median values of posteriors of 100 clusters with the same input parameters (open circles), we see a good agreement, suggesting that the assumption of Gaussian uncertainty of pixel values used in model fitting is valid. The uncertainty in the derived parameter values can in this case be judged from the "emcee" posterior samples of a single cluster.

We introduce the half-light radius, $r_{\mathrm{h}}$, in the analysis, since in numerous studies it is reported as cluster's size indicator. By analytically integrating Eq. 1, we get 


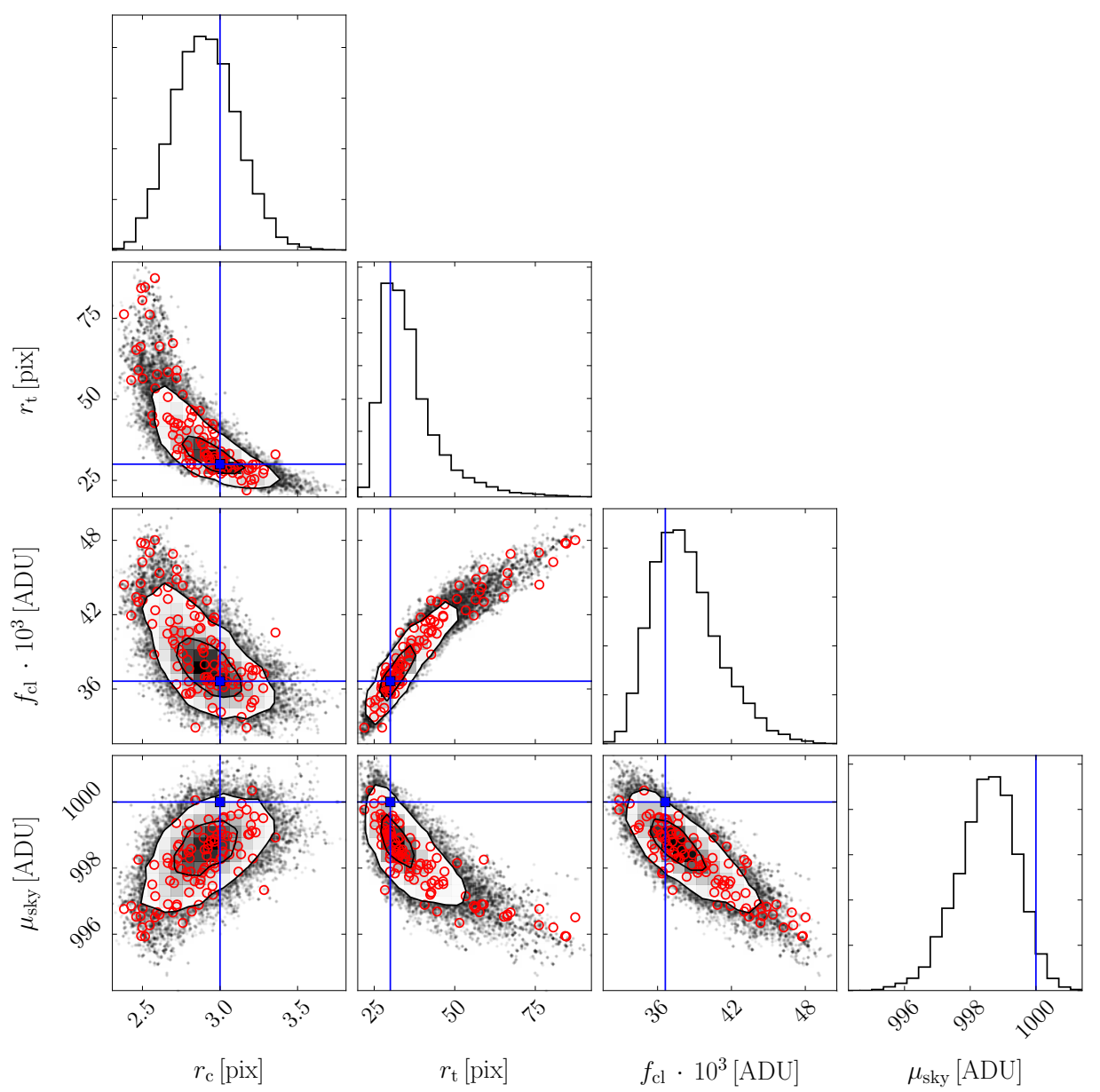

Fig. 3. Same as in Fig. 2, but for $S / N=50$. Note the shorter range of axis scales.

an expression for $r_{\mathrm{h}}$ as a function of $\left(r_{\mathrm{c}}, r_{\mathrm{t}}\right)$. This expression is solved numerically to yield $r_{\mathrm{h}}$. Once the posterior values of $r_{\mathrm{c}}$ and $r_{\mathrm{t}}$ are obtained by the "emcee", the posterior values of $r_{\mathrm{h}}$ are computed.

We compute median values of the parameter $\left(r_{\mathrm{c}}, r_{\mathrm{t}}, r_{\mathrm{h}}\right)$ posterior distributions for each node cluster from the model grid with sky background values of $\mu_{\text {sky }}$ $=10^{2}, 10^{3}$, and $10^{4} \mathrm{ADU}$ and $S / N=20,50,100,200$, and 500 (100 clusters per node). Then we compute the median and the standard deviation of each parameter per node (same $\mu_{\text {sky }}$ and $S / N$ ) and plot these values as a function of $S / N$ in Fig. 5. We see that for $S / N=20$ clusters, $r_{\mathrm{t}}$ is overestimated and $r_{\mathrm{c}}$ is underestimated because of a large degeneracy of these parameters as seen in Fig. 2. To constrain the cluster's half-light radius to be accurate to within $\sim 20 \%$, $S / N \sim 50$ is necessary in all realistic sky background level conditions $\left(10^{2}, 10^{3}\right.$, and $\left.10^{4} \mathrm{ADU}\right)$.

Bonatto \& Bica (2008) presented the results of their study on the accuracy of structural parameters of star clusters recovered from the radial profiles of light, 


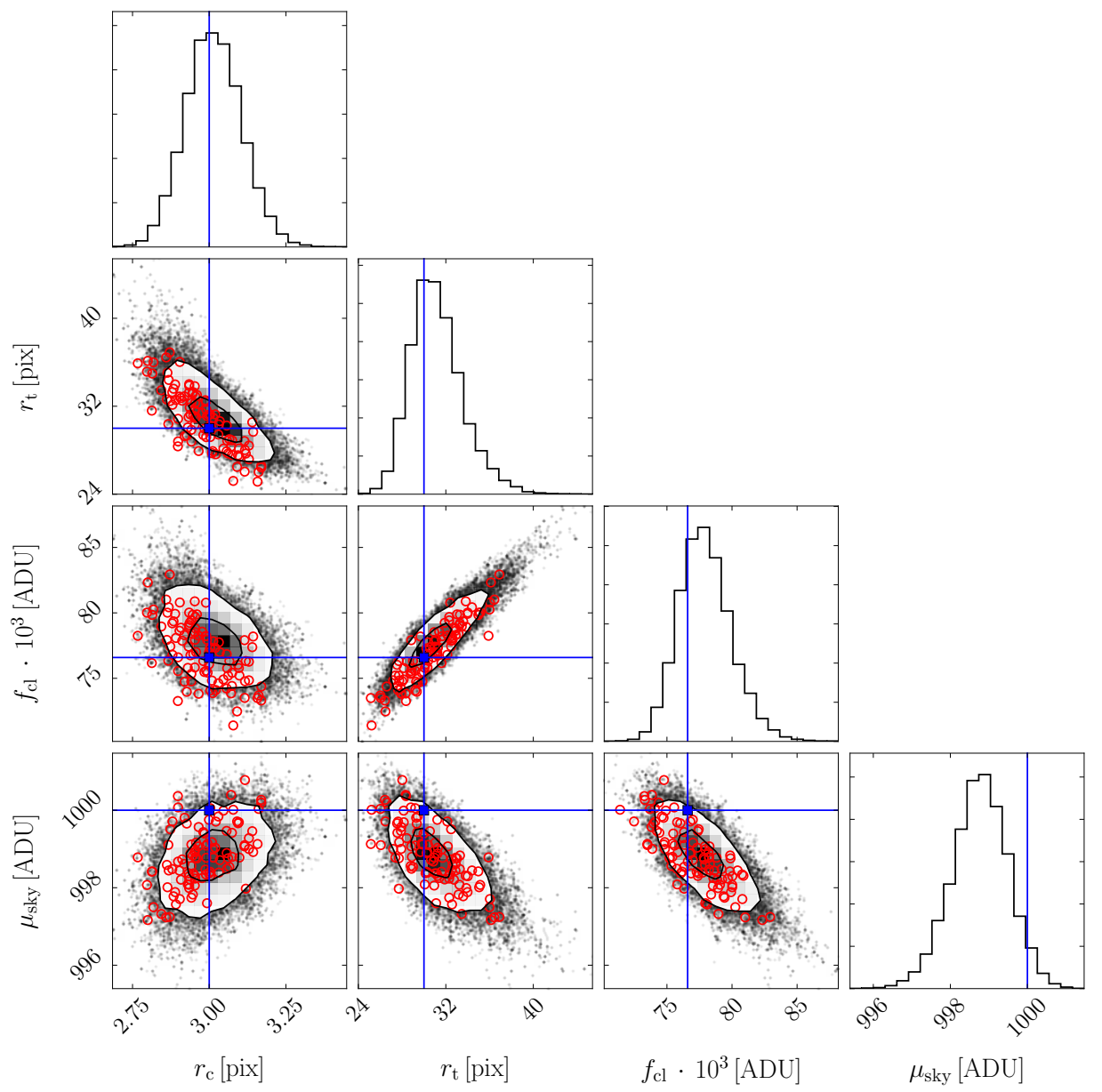

Fig. 4. Same as in Fig. 2, but for $S / N=100$. Note the shorter range of axis scales.

mass, and star counts and their dependence on photometric depth. They assumed that sky background level is known, subtracted it from the data and performed analysis of radial profiles only.

Carlson \& Holtzman (2001) performed an extensive analysis of the possibility of measuring King (1962) model parameters as a function of cluster's $r_{\mathrm{c}}, r_{\mathrm{t}}$, and signal to noise. They used a Levenberg-Marquard optimization algorithm, which provides the best-fit value of the model parameters. Only three free parameters were used to fit the model: position $(x, y)$, and $r_{\mathrm{c}}\left(r_{\mathrm{c}} / r_{\mathrm{t}}\right.$ was fixed). In this paper, we demonstrate the posterior distributions of all parameters that were used to generate the cluster's model, allowing also the sky background as a free parameter. By comparing the results for 100 clusters with the same parameters, we demonstrate that posterior distributions of a single cluster are a good indicator of true parameter uncertainty, if the image pixel uncertainty distribution is Gaussian. 


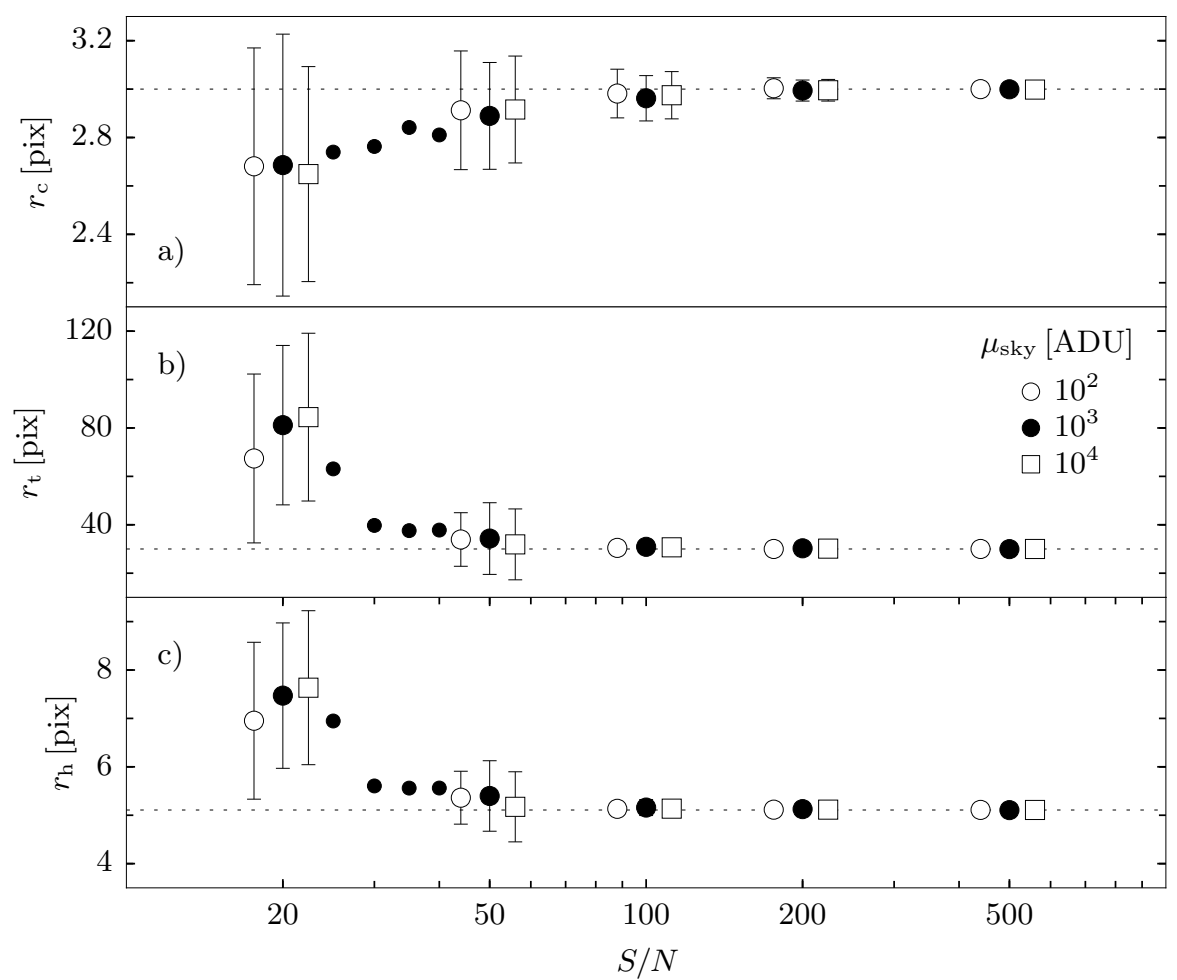

Fig. 5. Median and standard deviation of the recovered radii $r_{\mathrm{c}}, r_{\mathrm{t}}$, and $r_{\mathrm{h}}$ of 100 clusters per model grid node as a function of signal to noise, $S / N$. For the same $S / N$, symbols indicate different sky background, $\mu_{\text {sky }}=10^{2}$ (open circles), $10^{3}$ (large filled circles), and $10^{4} \mathrm{ADU}$ (squares). Horizontal dashed lines show true parameter values. Additional simulations at intermediate values of $S / N=25,30,35$, and 40 with $\mu_{\text {sky }}=$ $10^{3}$ ADU are shown by small filled circles.

\section{CONCLUSIONS}

Using the MCMC model fitting technique we have analyzed the influence of photometric signal to noise on the accuracy of derived structural parameters of unresolved star clusters. We have considered clusters with a King (1962) model surface brightness profile $\left(r_{\mathrm{c}}=3\right.$ pix,$r_{\mathrm{t}}=30$ pix $)$ and convolved it with a Gaussian $\mathrm{PSF}$ of FWHM $=3$ pix. We have used the "emcee" program to perform MCMC model fitting for the simulated grid of artificial clusters with various values of sky background and signal to noise.

We have demonstrated the degeneracies between the structural parameters, cluster's flux, and sky background level. By defining the photometric aperture containing $80 \%$ of the cluster's flux, we have found that $S / N \sim 50$ is critical to constrain tidal radius if the cluster resides in sky background of $10^{3} \mathrm{ADU}$. We have found that $S / N \sim 50$ is necessary in all realistic sky background level conditions $\left(10^{2}, 10^{3}\right.$, and $\left.10^{4} \mathrm{ADU}\right)$ to constrain the cluster's half-light radius to an accuracy better than $\sim 20 \%$. The presented technique can be applied to synthetic images simulating various observations of extragalactic star clusters. 
ACKNOWLEDGMENTS. We thank the referee for comments and suggestions which helped to improve the manuscript. This research was funded by a grant No. MIP-102/2011 from the Research Council of Lithuania.

\section{REFERENCES}

Bonatto C., Bica E. 2008, A\&A, 477, 829

Carlson M. N., Holtzman J. A. 2001, PASP, 113, 1522

Foreman-Mackey D., Hogg D. W., Lang D., Goodman J. 2013, PASP, 125, 306

King I. 1962, AJ, 67, 471

Newberry M. V. 1991, PASP, 103, 122 